\title{
Lesões de pele em recém-nascido: revisão integrativa
}

\author{
Skin lesions in newborns: integrative review
}

\author{
Fiama Kécia Silveira Teófilo ${ }^{1}$ Ana Valeska Siebra e Silva ${ }^{1} *$ Katherine Jerônimo Lima ${ }^{1}$ \\ Ana Paula Feles Dantas ${ }^{2}$ Valkenia Alves Silva² ${ }^{*}$ Tiago José Silveira Teófilo²
}

\section{RESUMO}

O objetivo é descrever e analisar as evidências cientificas acerca de lesões de pele em recém-nascido. Buscou-se produções científicas tendo como critérios de inclusão publicações período de 2012 a 2017, que correlacionassem os descritores "pele", "ferimentos e lesões" e "recém-nascido". As bases de dados pesquisadas foram PUBMED, WEB OF SCIENCE, LILACS, CINAHL e SCIELO. Ao analisarem-se os 10 estudos selecionados, foi consenso entre os autores que a produção de conhecimento científico referente à temática de lesões de pele em RN representa um eixo norteador para a assistência. A integridade da pele é de grande importância para a sobrevida do RN. Em virtude da sua atuação entre os órgãos internos e o meio externo, a pele atua como barreira contra infecção. Os fatores de risco para lesões de pele em RN estão associados aos procedimentos realizados no atendimento hospitalar. Conclui se que na literatura pesquisada, estudos que abordem os cuidados com a pele do RN diante de lesões ainda são escassos, portanto, faz-se necessário desenvolver novos estudos nesse sentido.

Palavras-chave: Pele; Ferimentos e lesões; Recém-nascido; Enfermagem.

\section{ABSTRACT}

The objective is to describe and analyze the scientific evidence about skin lesions in newborns. It was searched for scientific productions having as criteria of inclusion publications period from 2012 to 2017, that correlated the descriptors "skin", "injuries and lesions" and "newborn". The searched databases were PUBMED, WEB OF SCIENCE, LILACS, CINAHL and SCIELO. When analyzing the 10 selected studies, it was agreed among the authors that the production of scientific knowledge regarding the theme of skin lesions in NB, represents a guiding axis for the care. The integrity of the skin is of great importance for the survival of the newborn. Due to its action between the internal organs and the external environment, the skin acts as a barrier against infection. Risk factors for skin lesions in newborns are associated with procedures performed in hospital care. It is concluded that in the researched literature, studies that address the skin care of the newborn with lesions are still scarce, therefore, it is necessary to develop new studies in this sense.

Keywords: Skin; Wounds and injuries; Newborn; Nursing. 


\section{INTRODUÇÃO}

A pele é considerada o maior órgão do corpo humano e isola os componentes orgânicos do meio externo e presenta características distintas nas diferentes fases do ciclo de vida de um indivíduo. É constituída por três camadas distintas: epiderme, derme e hipoderme ou tecido subcutâneo, as quais exercem uma série de funcionalidades, entre elas: a proteção, a termorregulação, controle de infecção, imunovigilância e sensação tátil(1). No neonato a pele é caracterizada como delicada, fina e frágil, onde as reações fisiológicas e patológicas são frequentemente complexas, portanto, suas alterações são comuns nesse período. Estas características, associadas à imaturidade dos sistemas e à utilização de dispositivos médicos, imprescindíveis à sua sobrevivência, aumentam o risco de lesão da pele ${ }^{(2)}$.

Considera-se como lesão de pele qualquer achado incomum na superfície desta. As lesões podem ser primárias, quando representam um sinal inicial de processo patológico, ou secundárias, quando correspondem ao resultado de uma formação tardia ou trauma de uma lesão primária(3).

A North American Nursing Diagnosis Association apresenta fatores de risco para o desenvolvimento de lesões de pele, quais sejam: radiação, imobilização física, utilização de adesivos que arrancam pelos, pressão, contenção, distermias, umidade, substâncias químicas, excreções, secreções, medicações e extremo de idade ${ }^{(4)}$. Estudo de revisão identificou ainda duas outras causas para o desenvolvimento de lesões de pele em RN, que foram as injúrias relacionadas à manipulação inadequada do RN e ressecamento da pele ${ }^{(5)}$.

As lesões de pele em RN possuem importância para a saúde pública, especialmente no contexto hospitalar. Estimase que a incidência de lesões de pele em RN seja de $16 \%$, observando-se alta prevalência de $58 \%$. Sabe-se que as lesões de pele são uma das principais causas de prolongamento das internações dos neonatos, observando-se em média o prolongamento de 37,2 dias nas internações ${ }^{(6)}$.

Nesse contexto, a qualificação da assistência prestada pela equipe multiprofissional e a redução da morbimortalidade neonatal demandam ações específicas na prestação de cuidados peculiares ao recém-nascido (RN), que devem ser desenvolvidos de forma integral. Esse é um elemento básico para maior racionalidade e efetividade no processo de organização da atenção perinatal|(7).

No que diz respeito aos cuidados específicos de enfermagem, o objetivo da assistência é a condução do neonato à recuperação, adaptação e bem-estar, portanto, devem estar fundamentados em 
conhecimentos científicos e na autonomia do profissional de enfermagem ${ }^{(8)}$. Para 0 enfermeiro prestar a adequada assistência, faz-se necessário um aporte de evidências científicas que o auxiliem na escolha dos melhores produtos, técnicas, materiais e procedimentos. No entanto, apesar dos avanços em pesquisas na área, é necessário o desenvolvimento de pesquisas, principalmente com ênfase nos prematuros, população neonatal que apresenta especificidades devido à imaturidade fisiológica(9).

A partir do exposto, surge a seguinte pergunta de pesquisa: o que tem sido estudado recentemente sobre lesões de pele em recém-nascidos?

O exercício do cuidado de enfermagem em ambientes de cuidado intensivo a recémnascidos, tanto pré-termo quanto a termo, e o desafio da qualificação da assistência, foram os estimuladores para a produção da presente pesquisa.

Com intuito de responder à questão, observa-se que é necessária melhor compreensão desse evento, bem como a identificação de lacunas existentes no conhecimento, e de favorecer o aprimoramento da assistência prestada aos neonatos pela equipe de saúde e enfermagem com o intuito de prevenir as lesões de pele ou mesmo reduzir o impacto dessas sobre a saúde do RN. A revisão tem como objetivo descrever e analisar as evidências científicas que abordem lesões de pele em recémnascidos.

\section{MÉTODO}

Trata-se de uma revisão integrativa de literatura visando sintetizar os conhecimentos publicados de um determinado assunto ou temática, além de apontar lacunas que devem ser preenchidas com novos estudos em complementariedade à revisão. Segue-se o mesmo rigor metodológico de pesquisas originais, permitindo aos leitores subsídios para o avanço das práticas em saúde ${ }^{(10)}$. A presente revisão integrativa desenvolveu-se em seis etapas: 1) formulação da questão norteadora; 2) busca em bases de dados 3) seleção e categorização dos estudos; 4) avaliação dos estudos selecionados; 5) discussão e interpretação dos resultados; e 6) síntese do conhecimento com recomendações para a prática ${ }^{(11)}$.

A questão norteadora desta revisão foi: quais as evidências científicas acerca das lesões de pele em recém-nascidos? Foram critérios de inclusão: estudos publicados no período de 2012 a 2017, que analisaram lesões de pele em recém-nascidos, nas esferas da prevenção e da assistência pós desenvolvimento de lesões; considerou-se os estudos de níveis de evidência de I a IV que correspondem a meta análise ou meta síntese (nível I), estudo experimental ou ensaio clínico 
(nível II), estudo quase-experimental (nível III) e estudo não-experimental, como estudo de caso, revisão integrativa, pesquisa descritiva e estudo qualitativo (nível IV); que possuíam título e resumo indexados em bases de dados. Foram excluídos os artigos de reflexão e estudos metodológicos, ou que não abordavam diretamente lesões de pele, que fossem repetidos em mesmas bases de dados e que não fossem disponíveis online na íntegra.

A busca dos artigos foi realizada nas seguintes bases de dados: Literatura LatinoAmericana e do Caribe em Ciências da Saúde (LILACS), American National Library of Medicine (PubMed), Cumulative Index to Nursing and Allied Health Literature (CINAHL), Thomson Reuters Scientific (Web of Science) $e$ Scientific Electronic Library Online (SciELO). Para a busca nas bases de dados LILACS e SciELO, foram utilizados o Descritores em Ciências da Saúde (DeCS) "pele", "ferimentos e lesões" e "recém-nascido". Nas bases de dados PubMed, CINAHL e Web of Science, foram utilizados os descritores indexados no MeSH Terms em cruzamento: "Skin", "Wounds and Injuries" e "Infant, Newborn", com a utilização do operador booleano AND.

Foram localizados 205 artigos a partir da junção de todas as buscas. Todos os resumos foram acessados pela internet, resultando em 26 artigos desta pesquisa que atenderam aos critérios de inclusão, restando
10 ao final, ao considerar-se os critérios de exclusão. Definiu-se as informações a serem extraídas dos 10 estudos selecionados, tendo sido catalogadas em ficha bibliográfica organizada em uma planilha com os seguintes itens: autores e suas titulações, idioma em que foram publicados, país de realização das pesquisas, título, ano de publicação, palavraschave ou descritores, método, objetivos, periódico e base de dados. Buscou-se também identificar e discutir os principais resultados, conclusões e recomendações para a prática de Enfermagem, as sugestões de novas pesquisas e, por fim, as dificuldades apresentadas.

Ocorreu o preenchimento e análise crítica dos estudos selecionados, observando os aspectos metodológicos, o consenso entre os diferentes pesquisadores e suas divergências. Destaca-se que para essa etapa, após leitura exaustiva dos textos completos, foi possível destacar as seguintes categorias: (1) riscos para o desenvolvimento de lesões de pele e (2) mecanismos de tratamento de lesões de pele em recém-nascidos. Constituiuse na discussão e interpretação dos resultados, tendo sido elaboradas recomendações a partir das conclusões encontradas nesta revisão.

\section{RESULTADO}

Ao analisarem-se os dez estudos selecionados, observou-se que quatro deles utilizaram a abordagem quantitativa e três, a 
qualitativa. Os demais são revisões de literatura. Dos quatro quantitativos, três utilizaram-se de análise retrospectiva em ambiente de internação hospitalar. Outros ambientes de pesquisa incluíram ambulatório médico de puericultura e acompanhamento de pacientes com espinha bífida. $\mathrm{O}$ descritor "infant newborn" foi o que mais se repetiu, estrando em três dos estudos, seguido por "skin" $e$ "wounds and injuries", que apareceram em dois estudos cada um.

Dos instrumentos de coleta de dados utilizados, a observação e o registro das etapas do tratamento de lesões de pele em recém-nascidos estiveram presentes na metade dos estudos, enquanto que entrevistas semiestruturadas estiveram em dois estudos. O número total de participantes dos estudos que apresentaram lesões de pele foi 771. Profissionais de Enfermagem de uma Unidade de Terapia Intensiva Neonatal (UTIN) fizeram parte de um estudo como participantes. Percebeu-se quanto ao ano de publicação, que metade dos estudos selecionados foram publicados em 2013. O Quadro I apresenta a caracterização dos estudos da amostra.

Quadro 1. Caracterização dos estudos selecionados segundo identificação, objetivos, delineamento, sujeitos e instrumentos de coleta de dados. Fortaleza, CE, Brasil, 2018.

\begin{tabular}{|c|c|c|c|c|}
\hline $\begin{array}{c}\text { Refer } \\
\text { ência }\end{array}$ & Objetivo & $\begin{array}{c}\text { Delinea } \\
\text { mento }\end{array}$ & Sujeitos & $\begin{array}{c}\text { Instrumen } \\
\text { tos de } \\
\text { Coleta }\end{array}$ \\
\hline $\begin{array}{c}\text { Beall } \\
\text { et al. } \\
(2013)\end{array}$ & $\begin{array}{c}\text { Analisar } \\
\text { aspectos } \\
\text { de lesão }\end{array}$ & $\begin{array}{c}\text { Revisão } \\
\text { de } \\
\text { Literatur }\end{array}$ & $\begin{array}{c}\text { Trata-se } \\
\text { de } \\
\text { revisão }\end{array}$ & $\begin{array}{c}\text { Não } \\
\text { relatado }\end{array}$ \\
\hline
\end{tabular}

\begin{tabular}{|c|c|c|c|c|}
\hline (12) & $\begin{array}{c}\text { de pele } \\
\text { relacionad } \\
\text { os à } \\
\text { infiltração } \\
\text { e } \\
\text { extravasa } \\
\text { mento. }\end{array}$ & $a$ & & \\
\hline $\begin{array}{l}\text { Boyar } \\
\text { et al. } \\
(2014) \\
(13)\end{array}$ & $\begin{array}{c}\text { Apresenta } \\
\text { r uma } \\
\text { série de } \\
\text { casos } \\
\text { descreven } \\
\text { do o uso } \\
\text { de Mel de } \\
\text { grau } \\
\text { médico - } \\
\text { Mel de } \\
\text { Leptosper } \\
\text { mum } \\
\text { (Medihon } \\
\text { ey), para } \\
\text { tratament } \\
\text { o bem- } \\
\text { sucedido } \\
\text { de feridas } \\
\text { neonatais } \\
\text { de } \\
\text { cicatrizaçã } \\
\text { o lenta, } \\
\text { especifica } \\
\text { mente } \\
\text { úlcera de } \\
\text { pressão } \\
\text { de estágio } \\
\text { 3, ferida } \\
\text { esternal } \\
\text { deiscente } \\
\text { e } \\
\text { infectada } \\
\text { e ferida } \\
\text { de } \\
\text { espessura } \\
\text { completa } \\
\text { de uma } \\
\text { lesão de } \\
\text { extravasa } \\
\text { mento. }\end{array}$ & $\begin{array}{l}\text { Qualitati } \\
\text { va/ Série } \\
\text { de Casos }\end{array}$ & $\begin{array}{c}3 \\
\text { neonato } \\
\text { s } \\
\text { hospitali } \\
\text { zados } \\
\text { em } \\
\text { situação } \\
\text { de } \\
\text { tratame } \\
\text { nto. }\end{array}$ & $\begin{array}{l}\text { Observaçã } \\
\text { o e } \\
\text { registro } \\
\text { das } \\
\text { etapas do } \\
\text { tratament } \\
\text { o das } \\
\text { lesões } \\
\text { com uso } \\
\text { de } \\
\text { Medihone } \\
\text { y }\end{array}$ \\
\hline $\begin{array}{l}\text { Ferrei } \\
\text { ra et } \\
\text { al. } \\
(2013) \\
(14)\end{array}$ & $\begin{array}{c}\text { Analisar } \\
\text { os fatores } \\
\text { associado } \\
\text { s às lesões } \\
\text { de pele } \\
\text { em } \\
\text { recém- } \\
\text { nascidos e } \\
\text { lactentes } \\
\text { atendidos } \\
\text { no } \\
\text { ambulatór } \\
\text { io de } \\
\text { puericultu }\end{array}$ & $\begin{array}{c}\text { Quantita } \\
\text { tivo / } \\
\text { Estudo } \\
\text { transver } \\
\text { sal, } \\
\text { descritiv } \\
\text { o }\end{array}$ & $\begin{array}{c}105 \\
\text { crianças } \\
\text { menore } \\
\text { s de } \\
\text { dois } \\
\text { meses. }\end{array}$ & $\begin{array}{c}\text { Entrevista } \\
\text { s } \\
\text { estruturad } \\
\text { as e } \\
\text { exame } \\
\text { físico } \\
\text { direcionad } \\
\text { o à } \\
\text { inspeção } \\
\text { da pele. }\end{array}$ \\
\hline
\end{tabular}




\section{ARTIGO ORIGINAL}

\begin{tabular}{|c|c|c|c|c|}
\hline & $\begin{array}{c}\text { ra de um } \\
\text { hospital } \\
\text { universitá } \\
\text { rio em } \\
\text { Recife/PE. }\end{array}$ & & & \\
\hline $\begin{array}{c}\text { Gonza } \\
\text { lez } \\
(2013) \\
(15)\end{array}$ & $\begin{array}{c}\text { Discutir os } \\
\text { quatro } \\
\text { maiores } \\
\text { subtipos } \\
\text { de } \\
\text { epidermol } \\
\text { ysis } \\
\text { bullosa e } \\
\text { sua } \\
\text { associaçã } \\
\text { o com } \\
\text { caracterís } \\
\text { ticas extra } \\
\text { cutâneas. }\end{array}$ & $\begin{array}{l}\text { Revisão } \\
\text { de } \\
\text { Literatur } \\
\text { a }\end{array}$ & $\begin{array}{c}\text { Trata-se } \\
\text { de } \\
\text { revisão }\end{array}$ & $\begin{array}{c}\text { Não } \\
\text { relatado }\end{array}$ \\
\hline $\begin{array}{c}\text { Lund } \\
\text { (2014) } \\
(16)\end{array}$ & $\begin{array}{c}\text { Explorar } \\
\text { diferenças } \\
\text { na pele } \\
\text { neonatal } \\
\text { em } \\
\text { pacientes } \\
\text { em UTI e } \\
\text { o risco do } \\
\text { desenvolv } \\
\text { imento de } \\
\text { lesões } \\
\text { cutâneas } \\
\text { relacionad } \\
\text { as ao } \\
\text { adesivo e } \\
\text { selecionar } \\
\text { produtos } \\
\text { mais } \\
\text { adequado } \\
\text { s para os } \\
\text { resultados } \\
\text { desejados }\end{array}$ & $\begin{array}{l}\text { Revisão } \\
\text { de } \\
\text { Literatur } \\
\text { a }\end{array}$ & $\begin{array}{c}\text { Trata-se } \\
\text { de } \\
\text { revisão }\end{array}$ & $\begin{array}{c}\text { Não } \\
\text { relatado }\end{array}$ \\
\hline $\begin{array}{c}\text { Nandi } \\
\text { olo- } \\
\text { Anelo } \\
\text { ne et } \\
\text { al. } \\
(2014) \\
(17)\end{array}$ & $\begin{array}{c}\text { Relatar } \\
\text { experiênci } \\
\text { a na } \\
\text { gestão de } \\
\text { extravasa } \\
\text { mento } \\
\text { por } \\
\text { iatrogenia } \\
\text { a visão } \\
\text { terapêutic } \\
\text { a, ocorreu } \\
\text { entre os } \\
\text { recém- } \\
\text { nascidos. }\end{array}$ & $\begin{array}{l}\text { Quantita } \\
\text { tivo / } \\
\text { Descritiv } \\
\text { o e } \\
\text { Retrosp } \\
\text { ectivo }\end{array}$ & $\begin{array}{c}15 \\
\text { neonato } \\
\text { s } \\
\text { internad } \\
\text { os. }\end{array}$ & $\begin{array}{c}\text { Registros } \\
\text { de } \\
\text { consultas } \\
\text { e } \\
\text { hospitaliz } \\
\text { ações e } \\
\text { registros } \\
\text { de } \\
\text { pacientes. }\end{array}$ \\
\hline $\begin{array}{l}\text { Ottoli } \\
\text { ni et } \\
\text { al. } \\
\text { (2013) }\end{array}$ & $\begin{array}{l}\text { Document } \\
\text { ar a } \\
\text { incidência } \\
\text { de feridas }\end{array}$ & $\begin{array}{l}\text { Quantita } \\
\text { tivo / } \\
\text { Estudo } \\
\text { de }\end{array}$ & $\begin{array}{c}376 \\
\text { paciente } \\
\text { s com } \\
\text { espinha }\end{array}$ & $\begin{array}{c}\text { Observaçã } \\
\text { o e } \\
\text { registro } \\
\text { dos }\end{array}$ \\
\hline
\end{tabular}

\begin{tabular}{|c|c|c|c|c|}
\hline (18) & $\begin{array}{c}\text { para } \\
\text { informar } \\
\text { os } \\
\text { pacientes } \\
\text { e os pais } \\
\text { sobre } \\
\text { como o } \\
\text { risco de } \\
\text { ferida } \\
\text { varia ao } \\
\text { longo da } \\
\text { vida e em } \\
\text { relação ao } \\
\text { nível } \\
\text { neurológi } \\
\text { co } \\
\text { funcional. }\end{array}$ & Coorte & bífida & $\begin{array}{l}\text { principais } \\
\text { locais } \\
\text { acometid } \\
\text { os com } \\
\text { lesões de } \\
\text { pele em } \\
\text { pacientes } \\
\text { com } \\
\text { espinha } \\
\text { bífida e } \\
\text { tratament } \\
\text { o. }\end{array}$ \\
\hline $\begin{array}{l}\text { Rente } \\
\text { a et } \\
\text { al. } \\
(2013) \\
(19)\end{array}$ & $\begin{array}{l}\text { Abordar } \\
\text { terapia de } \\
\text { ferida por } \\
\text { pressão } \\
\text { negativa } \\
\text { em } \\
\text { lactentes } \\
\text { e } \\
\text { crianças. }\end{array}$ & $\begin{array}{l}\text { Quantita } \\
\text { tivo / } \\
\text { Retrosp } \\
\text { ectivo }\end{array}$ & $\begin{array}{c}270 \\
\text { neonato } \\
\text { s e } \\
\text { paciente } \\
\text { s } \\
\text { pediátri } \\
\text { cos. }\end{array}$ & $\begin{array}{l}\text { Observaçã } \\
\text { o e } \\
\text { registro } \\
\text { das } \\
\text { etapas do } \\
\text { tratament } \\
\text { o das } \\
\text { lesões. }\end{array}$ \\
\hline $\begin{array}{c}\text { Santo } \\
\text { s et al } \\
(2014) \\
(20)\end{array}$ & $\begin{array}{c}\text { Conhecer, } \\
\text { junto à } \\
\text { equipe de } \\
\text { enfermag } \\
\text { em, os } \\
\text { cuidados } \\
\text { necessári } \\
\text { os para } \\
\text { tratament } \\
\text { o de } \\
\text { lesões de } \\
\text { pele em } \\
\text { recém- } \\
\text { nascidos } \\
\text { internado } \\
\text { s em uma } \\
\text { Unidade } \\
\text { Neonatal. }\end{array}$ & $\begin{array}{l}\text { Qualitati } \\
\text { va / } \\
\text { Converg } \\
\text { ente } \\
\text { Assisten } \\
\text { cial }\end{array}$ & $\begin{array}{c}4 \\
\text { auxiliare } \\
\text { s de } \\
\text { enferma } \\
\text { gem, } 6 \\
\text { técnicos } \\
\text { de } \\
\text { enferma } \\
\text { gem e } 4 \\
\text { enferme } \\
\text { iras. }\end{array}$ & $\begin{array}{c}\text { Entrevista } \\
\text { s } \\
\text { semiestru } \\
\text { turadas }\end{array}$ \\
\hline $\begin{array}{l}\text { Slavin } \\
\text { et al } \\
(2012) \\
(21)\end{array}$ & $\begin{array}{c}\text { Apresenta } \\
\text { r } 2 \text { casos } \\
\text { de } \\
\text { Tumoral } \\
\text { Calcinosis } \\
\text { (TC) de } \\
\text { um } \\
\text { hospital e } \\
53 \\
\text { espécimes } \\
\text { ressecada } \\
\text { s de } 7 \\
\text { pacientes } \\
\text { com TC. }\end{array}$ & $\begin{array}{l}\text { Qualitati } \\
\text { va/ Série } \\
\text { de Casos }\end{array}$ & $\begin{array}{c}1 \\
\text { criança } \\
\text { e } 1 \\
\text { adolesc } \\
\text { ente }\end{array}$ & $\begin{array}{l}\text { Observaçã } \\
\text { o e } \\
\text { registros } \\
\text { histológic } \\
\text { os e } \\
\text { químicos. }\end{array}$ \\
\hline
\end{tabular}

Fonte: dados da pesquisa. 
Quanto aos objetivos expostos pelos pesquisadores, quatro basearam-se na preocupação em responder, com evidências científicas, problemas de maior incidência e prevalência em cuidados com a pele inerente ao serviço de saúde no qual se inserem. Dois estudos abordam meios diferentes de tratamento a lesões de pele existentes. Quatro foram revisões específicas para determinadas lesões, como advindas de Epidermolysis bullosa, Tumoral Calcinosis e Spina Bifida, assim como abordam diferentes lesões por infiltração e extravasamento.

Para a discussão dos resultados, foi possível construir duas categorias, sendo a primeira (1) riscos para o desenvolvimento de lesões de pele, na qual são revisados aspectos que levam a formação de lesões de pele e suas especificidades, como derivadas de infiltração e extravasamento; epidermolysis bullosa e sua associação com características extra-cutâneas; lesões relacionadas ao uso de adesivo em curativos em UTIN e; avalia o risco de formação de ferida ao longo da vida em relação a recém-nascidos com nível neurológico funcional comprometido.

A segunda categoria (2) mecanismos de tratamento de lesões de pele em recémnascidos, aborda aspectos que se relacionam com meios de tratamento de lesões já existentes, com a sugestão do uso de Medihoney em feridas de lenta cicatrização; analisa extravasamento por iatrogenia na visão terapêutica; aborda terapia de ferida por pressão negativa; cuidados de Enfermagem diante de lesões de pele e; tratamento histológico em casos de presença de células Tumoral Calcinosis.

\section{DISCUSSÃO}

As evidências científicas que abordam lesões de pele em RN mostraram que é necessário o desenvolvimento de novas tecnologias de prevenção e tratamento tanto em contexto hospitalar quanto comunitário. A integridade da pele é de grande importância para a sobrevida do RN. Em virtude da sua atuação entre os órgãos internos e o meio externo, a pele atua como barreira contra infecção. No entanto, no RN, a pele possui uma superfície contínua, fina, delicada e frágil, sendo extremamente suscetível a lesões por diferentes meios.

Os fatores de risco para lesões de pele em RN estão associados aos procedimentos realizados no atendimento hospitalar que levam a riscos de formação de lesões cutâneas e suas especificidades, existem outros fatores associados, como excesso do uso de produtos químicos e cosméticos na higienização corporal e na lavagem das roupas das crianças, que podem induzir a irritação na pele, bem como, picadas de insetos que podem ocasionar prurido e irritação local, a 


\section{ARTIGO ORIGINAL}

qual pode evoluir para lesões do tipo pápula e vesícula $^{(14)}$.

Na ocorrência de lesões cutâneas, há necessidade de ser avaliado o grau de complexidade destas, para distinguir os casos de curso benigno e os casos patológicos. Desta forma, deve-se observar o agente causador, o período de lesão, a profundeza, a zona corporal em que se encontra, a extensão e as características presentes, para que se possa estabelecer orientações e condutas dos profissionais e dos pais para tratamento ideal da lesão ${ }^{(20)}$.

Ressalta-se que todos os estudos selecionados apresentaram recomendações para a prática clínica assistencial. Dentro da categoria riscos para o desenvolvimento de lesões de pele, são fatores vesicantes de risco: $\mathrm{pH}<5$ ou $\mathrm{pH}>9$; aumento da osmolaridade; efeito direto de drogas e solubilidade. Estes fatores acarretam dano vascular celular, liberando radicais que atuam diretamente nos processos inflamatórios, com dano as membranas celulares e as células sanguíneas, levando a perda da integridade celular, tromboses, extravasamento de líquidos e necroses celulares, com danos aos tecidos. Associado a esses fatores, têm-se os as condições frágeis da pele dos recém-nascidos, como fragilidade venosa, realização de múltiplas punções e limitação de visibilidade venosa, bem como, as patologias inerentes à pele ${ }^{(12)}$.
Pesquisa realizada sobre Epidermólise Bolhosa (EB), doença do tecido conjuntivo que causa bolhas na pele, provocando fricção e fragilidade, evidenciou que sua severidade varia desde casos leves até fatais, ainda sem cura. Nesse estudo de revisão, os quatro tipos principais de EB são abordados: a EB Simples, EB Juncional, a EB Distrófica e Síndrome de Kindler são discutidas, enfocando as características extracutâneas associadas. A avaliação de um RN suspeito de ter EB, incluindo o diagnóstico e gestão, também é revisto(20).

Vale ressaltar, que a maioria dos procedimentos realizados no RN com EB pode traumatizar a pele, devendo serem adaptados de forma que diminuam esse risco. Considerase também que muitos $\mathrm{RN}$ são prematuros, o que aumenta ainda mais a fragilidade cutânea. As lesões de pele e mucosas nessa fase da vida podem predispor a infecções graves e potencialmente fatais, além de causar desidratação. O acometimento da pele e mucosa varia conforme o tipo de EB, mas de modo geral, os cuidados devem ser tomados para todo recém-nascido com suspeita da doença e o diagnóstico feito o mais breve possível ${ }^{(22)}$. Em relação ao cuidado da pele dos acometidos por EB, as melhores práticas clínicas, indicam para todas as idades, curativos não adesivos, com produtos à base de silicone, pois são úteis na redução da dor causada por traumatismo cutâneo ${ }^{(23)}$. 
Outro risco para a presença de lesões cutâneas são as causadas por adesivos medicinais, sendo um problema conhecido para pacientes de unidades de cuidados intensivos neonatais. A lesão cutânea relacionada ao adesivo para todas as populações de pacientes inclui problemas mecânicos, como descamação da pele e bolhas de tensão; reações de dermatite tais como dermatite de contato irritativa e dermatite alérgica; e outras complicações como maceração da pele e foliculite. $\mathrm{O}$ mais observado em pacientes neonatais é a descamação da pele ${ }^{(16)}$.

Os produtos adesivos são selecionados com base na finalidade pretendida, bem como na localização anatômica que o adesivo será anexado. Uma importante consideração para cada paciente é o tipo de dispositivo que está sendo protegido pelo adesivo. Diferentes produtos podem ser mais adequados para fixar dispositivos críticos tais como tubos endotraqueais, dispositivos de acesso vascular, tubos torácicos e, em alguns casos, sondas nasogástricas em comparação com um sensor de monitorização que tem de ser substituído várias vezes ao dia. A prevenção de lesões por adesivos deve ser feita utilizando-se menores produtos adesivos. A dificuldade do manejo terapêutico dessas lesões requer prevenção através do desenvolvimento de protocolos de monitoramento e educação permanente dos funcionários em prol de infusões práticas eficientes ${ }^{(16)}$.

O uso de adesivos ou coberturas com silicone suave são considerados menos agressivos à pele dos RN. Sua estrutura de silicone favorece menor risco, pois adere suavemente tanto pele a íntegra quanto a pele peri-lesional, melhora a adaptabilidade e promove conforto, contribuindo para diminuir com os agravos provocados pelo cuidado da pele do neonato. Em todos os casos, no entanto, é importante ressaltar a necessidade de observar a maturidade cutânea do neonato de acordo com as semanas de vida; a maturação da barreira da pele após parto prematuro, que requer em média 2 a 4 semanas, e são fatores que determinam a prática de cuidados ${ }^{(24)}$.

Estudos apontam que alguns cuidados de enfermagem podem contribuir para manutenção da integridade da pele do RN, tais como: mudança de decúbito, manutenção da pele limpa e seca, uso de hidrocolóides em proeminências osseas, otimização na coleta de exames, evitando assim repetidas punções, prevenção e tratamento de extravasamento e infiltração de soluções intravenosas, assim como, a identificação de lesões cutâneas comuns, tais como eritema, dermatite de fraldas, milium e hiperplasia de glâdulas sebaceas ${ }^{(26)}$.

Pesquisa realizada em uma Unidade Neonatal no Sul do Brasil revelou que existem 
dificuldades por parte dos profissionais, principalmente enfermeiros, na tomada de decisão da conduta para o tratamento e continuidade do cuidado da pele em RN. Citam que a ausência de padronização para procedimentos e uso adequado de produtos nos cuidados das lesões, as peculiaridades fisiológicas pertinentes ao $\mathrm{RN}$, como também insuficientes estudos sobre a temática, somam-se como obstáculos para efetivação de assistência de qualidade(20) ${ }^{(20) s a}$ forma, torna-se iminente a construção de um protocolo para padronização de condutas e prestação da assistência, baseada em conhecimentos científicos para um cuidado individualizado, almejando estabelecer diretrizes para reduzir o risco de mudanças nas condutas, proporcionando atendimento qualificado e seguro ao recém-nascido.

Em relação aos achados referentes aos mecanismos de tratamento de lesões de pele em recém-nascidos, utilizando-se terapia por pressão negativa como método de cuidado de feridas complexas, foi comprovada sua eficácia em estudo com 270 pacientes neonatais e pediátricos. O sistema de fechamento a vácuo aplica pressão de sucção negativa à base da ferida através de uma esponja porosa selada por uma cobertura aderente, respeitando a quantidade de pressão ideal para cada faixa etária. Se a terapia por pressão negativa fosse posta sobre as vísceras ocas, colocava-se uma gaze não aderente revestida de petróleo antes de aplicar a esponja em colagem $\operatorname{adesiva}^{(19)}$.

Reposições de fluidos eram realizadas em casos de perda de líquido da ferida em quantidade significativa. Foram analisadas características como gênero, idade, comorbidades associadas e nutrição. Classificaram-se as feridas de acordo com a etiologia (isto é, trauma, criadas cirurgicamente, congênitas, queimaduras, úlceras de pressão e enxertos), idade (aguda versus crônica) e localização anatômica. Observaram-se também quaisquer características únicas do local tratado, tais como a presença de osso, intestino, tendão ou nervo. Foram analisados também detalhes do tratamento relativos a duração e frequência de alterações ${ }^{(16)}$.

O uso da terapia por pressão negativa promove cicatrização por secundária ou terciária intenção. Comprovou-se que reduz o edema proveniente das feridas, favorecendo a granulação do tecido em formação, aumentando a perfusão e removendo exsudatos e material infeccioso. Embora a Pediatric Medical Device Safety reconheça que, por vezes, os dispositivos cirúrgicos não estão imediatamente disponíveis para população, e com as vantagens citadas do uso da terapia por pressão negativa, o uso em neonatos e lactentes tem recomendações de monitoramento mais intensivo, porque ocorreram complicações em cinco pacientes 
do estudo, com formação de fístula enteroatmosférica, desagregação da pele do adesivo, uma esponja retida e infecção de início recente na ferida ${ }^{(19)}$.

No que diz respeito à pesquisa acerca das injúrias causadas na pele do $\mathrm{RN}$ por acidentes perfusionais por iatrogênia, em 15 casos de recém-nascidos, entre janeiro de 2010 e dezembro de 2012, evidenciou-se que acidentes dessa natureza são sérias complicações frequentes que ocorrem durante uma infusão na corrente sanguínea, com fins diagnóstico ou terapêutico, em linhas venosas periféricas ou centrais. Se visualizados tardiamente, são fontes de sequelas funcionais $^{(17)}$.

O soro de cloreto de cálcio a $10 \%$ e glicose foram encontrados em todos os casos. Os membros superiores foram os mais afetados, com nove lesões e seis nos membros inferiores. As superfícies dorsais dos pés e das mãos foram encontradas respectivamente em seis e cinco casos. Duas lesões localizaram-se na região posterior do antebraço e cotovelo. 0 tratamento utilizou-se de seis curativos alcoólicos. A cirurgia foi tratamento secundário em quatro casos, combinada com excisão de enxerto de pele ${ }^{(17)}$.

No que concerne à utilização do cateter periférico intravenoso como dispositivo de acesso vascular mais utilizado para a administração de medicamentos em recémnascidos hospitalizados, observou-se que $95 \%$ desses cateteres são removidos devido a complicações. A infiltração e o extravasamento são as complicações mais destrutivas para a pele frágil do recémnascido. Verificou-se aspectos da lesão por infiltração e extravasamento, a partir do nível celular, onde se é discutido o papel dos vesicantes na lesão vascular e seu papel desencadeando a inflamação, seguido por uma revisão abrangente dos vesicantes e seu mecanismo de lesão, por pH, osmolalidade ou composição química, e uma visão geral dos conhecimentos e ações das enfermeiras de UTIN para prevenir a infiltração(12).

O uso de um algoritmo baseado em evidências foi desenvolvido em um hospital infantil para minimizar lesões causadas por extravasamentos através de tratamento direcionado e imediato. Esse algoritmo traça marcadores de identificação de lesão, desde sua formação até as formas de tratamento. 0 uso de abordagens não-farmacológicas para tratar a lesão é um primeiro passo para limitação de danos. Para certas lesões induzidas por vesicantes, tratamento farmacológico com hialuronidase, em particular, mostrou-se eficiente para limitar a lesão. Se ineficaz, ou se a lesão é extensa, tratamento cirúrgico pode ser necessário e um cirurgião plástico pode ser consultado ${ }^{(12)}$.

Dentre a seleção de produtos que são mais adequados para fixar dispositivos críticos, um nível mais alto de produto de 
adesão, geralmente acrilato ou hidrocolóide, com um apoio mais forte, pano ou seda é selecionado. Os dispositivos de acesso vascular são protegidos com coberturas de poliuretano que contenham adesão de acrilato de modo que o local de inserção permaneça claramente visível. A fita Micropore é frequentemente utilizada pela facilidade de uso, pois pode ser facilmente rasgada em pequenos pedaços, reduzindo a quantidade de fita em contato com a pele. Fitas de silicone e produtos adesivos estão se tornando mais comuns e possuem qualidades promissoras, principalmente para a gestão de queimaduras $^{(12-26-27)}$.

Estudos iniciais têm apontado melhor resposta para prevenção de eritema com o uso de fitas adesivas de silicone do que com outros tipos de fita. Além disso, observou-se que o desconforto, a remoção de queratina e de pelos foi significativamente menor quando comparada a fita de silicone em comparação outras fitas $^{(28)}$. A descamação epidérmica da pele neonatal pode ser minimizada com o uso de curativos com adesivos à base de silicone $\operatorname{macio}^{(29)}$.

Embora os princípios gerais de cicatrização de feridas sejam semelhantes em crianças e adultos, existem diretrizes clínicas limitadas para direcionar a escolha de produtos específicos para tratamento de feridas no tratamento de feridas em crianças $^{(30)}$. É pertinente que os cuidados que preservem a integridade dos recém-nascidos sejam prioritários durante a internação em UTIN. Um cuidado que previna lesões de pele e infecções oriundas destas lesões, mantendo a pele íntegra e proporcionando conforto. As informações geradas nesse estudo podem ser utilizadas pelos profissionais como subsídios para a prática profissional, consolidando suas estratégias de cuidar da pele do recémnascido.

\section{CONCLUSÃO}

$\mathrm{Na}$ literatura internacional, estudos que abordem os cuidados com a pele do recémnascido tanto na prevenção, quanto diante de lesões, ainda são escassos. A presente revisão integrativa, realizada com o objetivo de analisar as publicações científicas que abordaram lesões de pele em $\mathrm{RN}$, reforça a necessidade de novos estudos que possam demonstrar, de maneira enfática e eficaz, mecanismos mais variados de prevenção e tratamento de lesões de pele nessa população. Essa necessidade mostra-se muito mais importante quando levados em consideração os fatores de risco mais comuns encontrados principalmente nas UTIN, onde os cuidados prestados são mais invasivos.

Destaca-se a equipe de Enfermagem como principal responsável pelo manuseio dos recém-nascidos nestas unidades, e cuidados específicos desta categoria com a pele são necessários. Essa síntese do conhecimento, 
trazido pelos estudos incluídos nesta revisão, reforça a importância das pesquisas para fundamentar a prática da enfermagem e para estimular novos estudos na compreensão da necessidade de se cuidar da pele do recémnascido, reduzindo a iatrogenia, e realizar tratamento adequado das lesões desenvolvidas.

A compreensão da importância da prevenção é primordial para que se evitem lesões. Observou-se que é necessário que a equipe multiprofissional trabalhe levando em consideração o uso de diferentes tipos de adesivos, coberturas de curativos, e outras tecnologias de tratamento, além de mecanismos técnicos eficazes para a prevenção e tratamento de lesões. Acredita-se que a principal limitação desta revisão é, portanto, o pequeno alcance quantitativo da produção científica internacional, sendo necessário ampliação das buscas, e a construção de novos estudos, especialmente revisões sistemáticas que abordem o $\mathrm{RN}$ e os riscos relacionados à integridade de sua pele em diferentes contextos de assistência à saúde.

\section{REFERÊNCIAS}

1. Eming SA, Martin P, Tomic-Canic M. Wound repair and regeneration: Mechanisms, signaling, and translation. Sci Transl Med. [Internet]. 2014 [acesso em 12 dez 2017]. 6(265): 265sr6. Disponível em: https://www.ncbi.nlm.nih.gov/pubmed/25473 038.
2. Martins COA; Curado MAS. Escala de Observação do Risco de Lesão da Pele em Neonatos: validação estatística com recémnascidos. Rev Enferm Referência. [Internet]. 2017 [acesso em 15 ago 2017]; 13 (4): 43-52. Disponível em: http://www.scielo.mec.pt/pdf/ref/vserlVn13/ serIVn13a05.pdf.

3. Potter, PA. et al. Fundamentos de enfermagem. 8. ed. Rio de Janeiro: Elsevier, 2013.

4. North American Nursing Diagnosis Association. Diagnóstico de enfermagem da NANDA: definições e classificação 2015-2017. 10. ed. Porto Alegre: Artmed, 2015.

5. Nascimento RR, Landim TMA. Cuidados de Enfermagem na prevenção de lesões de pele em recém-nascido prematuro. Rev Eletrôn Atual Saúde. [Internet]. 2016 [acesso em 06 ago 2017]; 4(4): 66-73. Disponível em: http://atualizarevista.com.br/article/cuidados -de-enfermagem-na-prevencao-de-lesoes-depele-no-recem-nascido-prematuro-v-4-n-4/.

6. Migoto MT, Souza SNDH, Rosetto EG. Skin lesions of newborns in a neonatal unit: descriptive study. Online Braz J Nurs. [Internet] 2013 [acesso em: 14 mai 2017]; 12(2): 377-92. Disponível em: <http://www.objnursing.uff.br/index.php/nur sing/article/view/4042>.

7. Melo RCJ, Souza IEO, Paula CP. Enfermagem neonatal: o sentido existencial do cuidado na Unidade de Terapia Intensiva. Rev Bras Enferm. [Internet] 2013 [acesso em 12 mai 2017]; 66(5): 656-662. Disponível em: http://www.scielo.br/scielo.php?script=sci_ar ttext\&pid=S0034-71672013000500003.

8. Moraes-Filho IM, et al. Checklist do recémnascido: principais diagnósticos de enfermagem mediante intercorrências e susceptibilidade das mesmas no neonatal. Rev Cient Sena Aires. [Internet] 2017 [acesso em 22 de Agosto de 2017]; 6(1): 30-48. Disponível em: 


\section{ARTIGO ORIGINAL}

http://revistafacesa.senaaires.com.br/index.p hp/revisa/article/view/276.

9. Elzer HE. Bathing basics: how clean should neonates be? Adv Neonatal Care. [Internet]. 2013 [acesso em: $31 \mathrm{dez}$ 2017]; 13(3):188-9. Disponível em: http://doi.org/10.1097/ANC.0b013e318293d5 ee.

10. Soares $C B$, et al. Integrative review: concepts and methods used in nursing. Rev Esc Enferm USP. [Internet]. 2014 [acesso em 12 jan 2018]; 48(2):335-345. Disponível em: http://www.scielo.br/scielo.php?script=sci_ar ttext\&pid=S0080-62342014000200335.

11. Ganong LH. Integrative reviews of nursing research. Res Nurs Health. [Internet] 1987 [acesso em 10 dez 2017]; 10(1)1-11. Disponível em: https://www.ncbi.nlm.nih.gov/pubmed/36443 66.

12. Beall V, et al. Neonatal Extravasation: An Overview and Algorithm for Evidence-based Treatment. Newborn and Infant Nursing Reviews. [Internet] 2013 [acesso em 12 mai 2017]; 13(4):189-195. Disponível em: https://www.sciencedirect.com/science/articl e/pii/S1527336913000986.

13. Boyar V. et al. Clinical experience with Leptospermum honey use for treatment of hard to heal neonatal wounds: case series. J Perinatol. [Internet] 2014 [acesso em 12 mai 2017]; 34(2):161-3. Disponível em: https://www.ncbi.nlm.nih.gov/pubmed/24476 663.

14. Ferreira AS, et al. Lesões de pele em recém-nascidos e lactentes. Rev Enferm UERJ. [Internet]. 2013 [acesso em 13 mai 2017]; 21(2):208-215. Disponível em: http://www.facenf.uerj.br/v21n2/v21n2a12.p df.

15. Gonzalez, ME. Evaluation and treatment of the newborn with epidermolysis bullosa. Semin Perinatol. [Internet] 2013 [acesso em
01 jun 2017]; 37(1):32-39. Disponível em: https://www.ncbi.nlm.nih.gov/pubmed/23419 761.

16. Lund, C. Medical Adhesives in the NICU. Newb Inf Nurs Rev. [Internet]. 2014 [acesso em 12 mai 2017]; 14(4):160-165. Disponível em:

https://www.sciencedirect.com/science/articl e/pii/S1527336914000877.

17. Nandiolo-Anelone $K R$, et al. Les accidents d'extravasation perfusionnelle chez le nouveau-né: une expérience de 15 cas. Chirurgie de la main. [Internet] 2014 [acesso em 01 jun 2017]; 33(1):44-50. Disponível em: https://www.sciencedirect.com/science/articl e/pii/S1297320313003880.

18. Ottolini K. et al. Wound care challenges in children and adults with spina bifida: an opencohort study. J Pediatr Rehabil Med. [Internet] 2013 [acesso em 14 mai 2017]; 6(1):1-10. Disponível em: https://hsrc.himmelfarb.gwu.edu/cgi/viewcon tent.cgi?article $=1100 \&$ context $=$ smhs_peds_fa cpubs.

19. Rentea RM. Negative pressure wound therapy in infants and children: a singleinstitution experience. J Surg Res. [Internet] 2013 [acesso em 02 jun 2017]; 184(1):658-64. Disponível em: https://www.ncbi.nlm.nih.gov/pubmed/23768 766.

20. Santos SV, Costa R. Prevenção de lesões de pele em recém-nascidos: conhecimento da equipe de enfermagem. Texto Contexto Enferm. [Internet]. 2015 [acesso em 06 ago 2017]; 24 (1): 731-39. Disponível em: http://www.scielo.br/pdf/tce/v24n3/pt_01040707-tce-24-03-00731.pdf. 


\section{ARTIGO ORIGINAL}

21. Slavin RE, Wen J, Barmada A. Tumoral calcinosis-a pathogenetic overview: a histological and ultrastructural study with a report of two new cases, one in infancy. Int J Surg Pathol. [Internet] 2012. [acesso em 06 jun 2017]; 20(5) 462-473. Disponível em: https://www.ncbi.nlm.nih.gov/pubmed/22614 164.

22. Pitta AL, Magalhães RP, Silva JC. Epidermóclise bolhosa congénita: importancia do cuidado de enfermagem. Rev Cuid [Internet] 2016 [acesso em 20 jan 2018]; 10(2):201-208. Disponível em: http://www.webfipa.net/facfipa/ner/sumario s/cuidarte/2016v2/201-208.pdf.

23. Goldschneider KR, et al. Pain care for patients with epidermolysis bullosa: best care practice guidelines. BMC Medicine [Internet]. 2014 [acesso em 12 mar 2018]; 12:178. Disponível em: https://bmcmedicine.biomedcentral.com/arti cles/10.1186/s12916-014-0178-2.

24. Mendes, L, Telles R, Ettinge S, Barbosa T. O uso do silicone suave na pele do neonato. Enferm Atual. [Internet] 2012 [acesso em 15 mar 2018]; 1(4). Disponível em: http://revistaenfermagematual.com.br/0101.php.

25. Schaefer TIM; Naidom AM; Neves ET. Cuidados com a pele do recém-nascido internado em unidade de terapia intensiva neonatal: revisão integrativa. Rev On Pesq. [Internet] 2016. [ acesso em 20 nov 2017]; 8(4): 5156-5162. Disponível em: http://www.seer.unirio.br/index.php/cuidado fundamental/article/view/3791.

26. Mir $M$, et al. Synthetic polymeric biomaterials for wound healing: a review. Prog Biomater. [Internet]. 2018 [acesso em 01 mar 2018]; 7: 1-21. Disponível em: https://www.ncbi.nlm.nih.gov/pubmed/29446 015.

27. Dreifke $M B$, Jayasuriya $A A$, Jayasuriya $A C$. Current wound healing procedures and potential care. Mater Sci Eng C Mater Biol Appl. [Internet]. 2015 [acesso em $20 \mathrm{mar}$ 2018]; 48: 651-662. Disponível em: https://www.ncbi.nlm.nih.gov/pmc/articles/P MC4443476/.

28. Grove GL, et al. Randomized Comparison of a Silicone Tape and a Paper Tape for Gentleness in Healthy Children. J Wound Ostomy Continence Nurs. [Internet]. 2014 [acesso em 20 mar 2018]; (1):40-8. Disponível em:

https://www.ncbi.nlm.nih.gov/pubmed/24240 641.

29. King A, et al. Dressings and Products in Pediatric Wound Care. Adv Wound Care. [Internet]. 2014 [acesso em 31 mar 2018]; 3(4): 324-334. Disponível em: https://www.ncbi.nlm.nih.gov/pmc/articles/P MC3985526/.

30. Sood A, Granick MS, Tomaselli NL. Wound Dressings and Comparative Effectiveness Data. Adv Wound Care. [Internet]. 2014 [acesso em 01 abr 2018]; 3(8): 511-529. Disponível em: https://www.ncbi.nlm.nih.gov/pubmed/25126 472. 